\title{
Sub-network Based Hierarchical Segment Restoration in MPLS Network
}

\author{
Hae-Joon Shin ${ }^{1}$, Sang-Heon Shin ${ }^{2}$, and Young-Tak Kim ${ }^{1}$ \\ 1 ANT Lab, Yeungnam University, \\ Gyeongsan Gyeongbuk 712-749, Korea \\ \{fisher, ytkim\}@yu.ac.kr \\ http://antl.yu.ac.kr \\ 2 Advanced Network Technologies Division(ANTD), \\ National Institute of Standards and Technology(NIST), \\ Gaithersburg, MD 20899, USA \\ shshin@nist.gov
}

\begin{abstract}
In this paper, we propose a segment restoration scheme based on network partitioning to enhance the restoration performance and to manage network efficiently. The proposed restoration scheme divides a large network into several small sub-networks. Since most faults can be restored in a partitioned sub-network or AS(Autonomous System), restoration time is reduced obviously. In this paper, we compare and analyze restoration performance according to the size of sub-networks and restoration schemes. From simulation results, the proposed restoration scheme has high restoration performance compared with other restoration schemes.
\end{abstract}

\section{Introduction}

A fault in the high-speed network, such as WDM(Wavelength Division Multiplexing) network and MPLS(Multi-Protocol Label Switching) [1], [2] may cause the massive data losses and degrade the quality of service severely. Therefore fast fault restoration function is an essential requirement in high-speed transport networks.

Any faulty or disconnected working path will severely degrade the end-to-end packet transmission and end-to-end quality of service. So any faulty link/node should be verified as soon as possible, and the user traffic should be rerouted quickly to minimize the possible packet loss. So, fast restoration is essential to increase the reliability of the quality of services of real time multimedia applications. For fast restoration, fast fault detection and fault notification functions are required.

Legacy restoration schemes on mesh network are based on link restoration or path restoration. Link restoration has the advantage of restoration speed but is poor at resource utilization. On the other hand, path restoration is good at resource utilization but poor at restoration speed. So various segment-based restoration schemes [3], [4], [5] are issued to enhance restoration performance. 


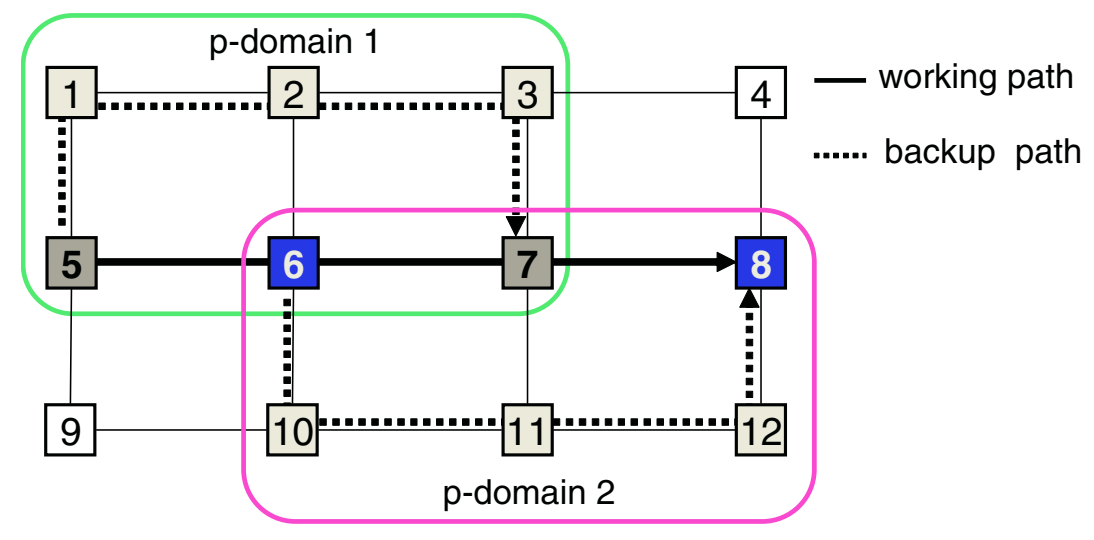

Fig. 1. Shortest Leap Shared Protection (SLSP)

In our previous research [6], [7], we describe basic concepts and principles of segment restoration. In this paper, we propose an enhanced segment restoration scheme based on network partitioning. We also propose provisioning methods of the inter segment backup path and an efficient network partitioning rules for enhanced restoration performance. We analyze restoration performance according to the size of partitioned sub-networks and compare with link-based restoration and path-based restoration.

The rest of this paper is organized as follows. In Section 2, we describe related approaches. In Sections 3 and 4, we propose segment restoration scheme and evaluate its restoration performance, respectively. And we make the conclusion in Section 5.

\section{Related Work}

\subsection{SLSP (Shortest Leap Shared Protection)}

SLSP has been proposed to enhance the link- and path-based protection; it partitions an end-to-end working path into several equal-length and overlapped segments [3]. Each segment assigns a protection domain for restoration after a working path is selected. The overlap between adjacent protection domains is designed to protect any boundary node failure along a working path. The SLSP scheme reduces restoration time obviously. In Fig. 1, working path 5-67-8 is divided into segments 5-6-7 and 6-7-8, and their backup paths are 5-12-3-7 and 6-10-11-12-8. Especially when domain size is 3(a segment consists of three nodes), the restoration time becomes the shortest. But, the SLSP should divide each logical path in a network into several domains(segments) and provide protection domain(segment) for each domain regardless of the hierarchical subnetwork configuration of the given network. Therefore, when the number of path in a network increases, the processing complexity also increases. 


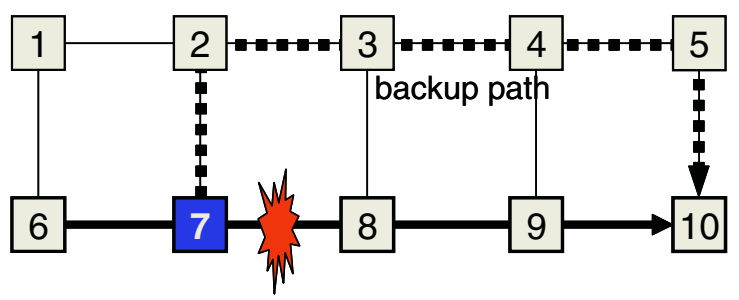

Fig. 2. Sub-path restoration

\subsection{Sub-path Restoration}

Sub-path restoration [4] patches backup path with a node that detects fault occurrence. When a fault occurs at Link 7-8 as shown in Fig. 2, the upstream node (Node 7 ) of the failed path does not send an alarm to the source node(Node 6) of the disrupted path; instead, it tries to patch a path by sending a setup message to the destination node(Node 10). Meanwhile, the downstream node(Node 8) sends a teardown message to the destination node(Node 10) of the working path. Since a backup path 2-3-4-5-10 is patched with Node 7, so user traffic is switched at Node 7 and rerouted along 6-7-2-3-4-5-10.

The sub-path restoration scheme reduced restoration time compared with the path restoration scheme relatively. When a fault occurs around an egress node, restoration time is reduced obviously, but when a fault occurs around an ingress node, restoration time similar with the path restoration scheme. Since a backup path is provisioned after a fault occurs, restoration performance is lower than other restoration schemes that use backup path restoration scheme.

\subsection{PROMISE (Protection with Multi-segment)}

The basic idea of PROMISE [5] is to divide an active path or AP(along which a survivable connection is established) into several possible overlapping active segments (AS) and then to protect each AS with a detour called backup segment or BS(instead of protecting the AP as a whole as in path protection schemes). The BS starts and ends at the same two nodes as the corresponding AS [4].

In Fig. 3, an active path is divided three active segments (AS1, AS2 and AS3) and each active segment has a backup segment (BS1, BS2 or BS3) for

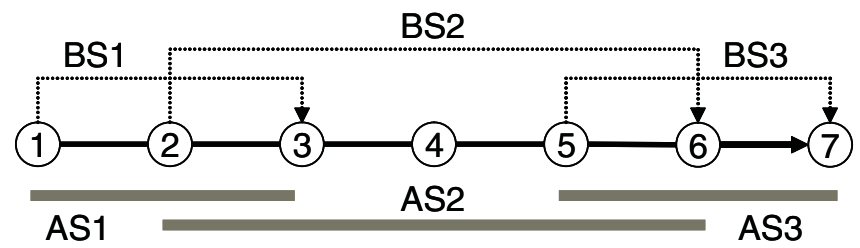

Fig. 3. Illustration of PROMISE 


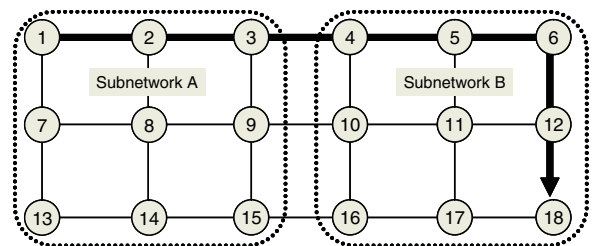

(a) Two sub-networks

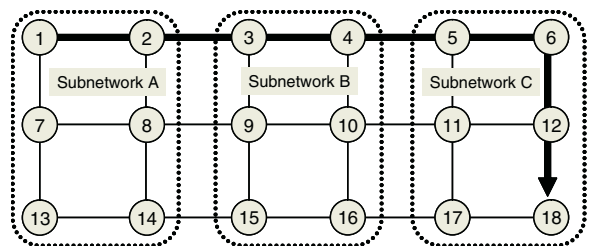

(b) Three sub-networks

Fig. 4. Network partitioning

protection respectively. Fault restoration speed is faster than legacy restoration schemes, because a fault can be restored in each segment. The basic idea of path dividing is similar with SLSP. SLSP divides an active path into several equal length segments, but in the PROMISE, the length of active segments is not equal.

\section{Proposed Segment Restoration}

\subsection{Principle of Proposed Segment Restoration}

The proposed segment restoration scheme is based on physical network partitioning as shown in Fig. 4. In Fig. 4(a), a network is divided into sub-network A and sub-network B, and an end-to-end path is divided into two segments by physical network partitioning automatically: 1-2-3 and 4-5-6-12-18. So a segment can be a part of an end-to-end whole path. The longest segment is an end-to-end whole path such as 1-2-3-4-5-6-12-18 and a shortest segment is a link such as link 1-2, or link 3-4 as shown in Fig. 4(b). The length of a segment is depends on the number of sub-networks.

To apply the proposed segment restoration scheme to real networks, we should consider two kinds of faults: intra sub-network fault and inter subnetworks faults as shown in Fig. 5. In our research, the detail of two sub-network faults and protection schemes is described.

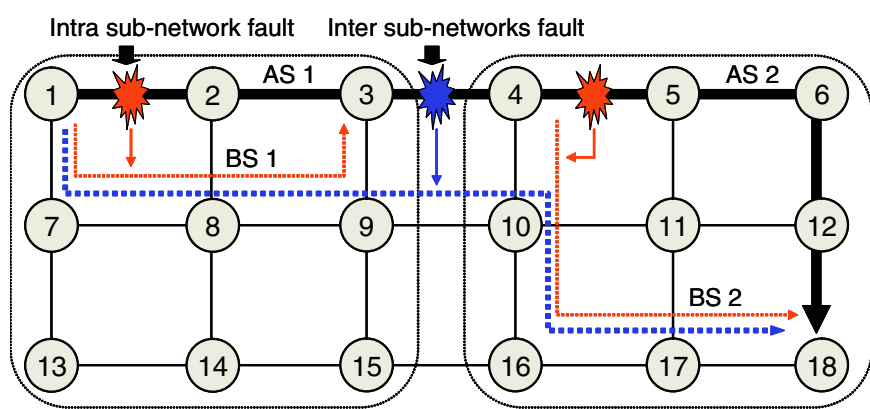

Fig. 5. Intra sub-network fault vs. inter sub-networks fault 


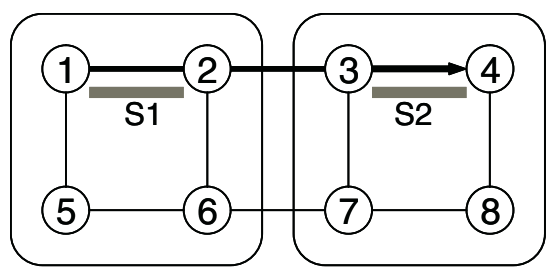

(a) Network based partitioning

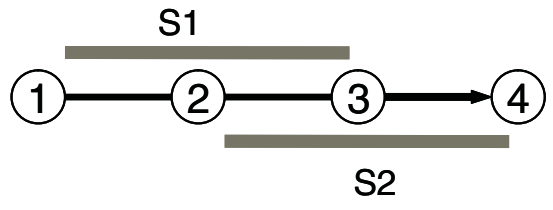

(b) Logical path based partitioning

Fig. 6. Path partitioning methods

\subsection{Path Partitioning vs. Network Partitioning}

Segment restorations are based on path partitioning that is achieved by logical path partitioning and physical network partitioning as shown in Fig. 6. The proposed segment restoration scheme divides a physical network to several subnetworks as shown in Fig. 6(a), while the other approaches [3], [5] are based on logical path partition as shown in Fig. 6(b).

Advantages of logical path based partitioning are: (1) length of segment can be adjusted according to the restoration strategy. The length of segment is very important factor to determine restoration speed. (2) It can provide steady restoration time to fix the segment length by NMS(Network Management System) and so on. Shortcomings are (1) who and how divides a path into several segments? Partitioning is achieved by per-path, so it makes network operation complex. (2) In a network which consists of several AS(Autonomous System) as shown in Fig. 7, if a path is established through several AS, it is impossible or difficult to divide the path into several segments.

The advantages of network-based partition are: (1) a path is divided into several segments by sub-networks automatically. (2) In a network where consists of several AS, when a path is established through several ASs, path partitioning is achieved by AS automatically. So it is easy to apply to various networks without modification of restoration method. (3) In network management perspective, it is easy and efficient to manage small size network rather than large size network. However, it also has shortcoming that proposed segment restoration provides two

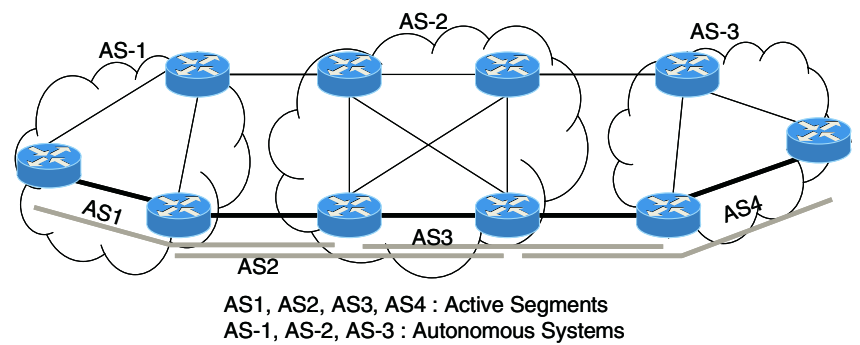

Fig. 7. Network which consists of three AS(Autonomous System) 


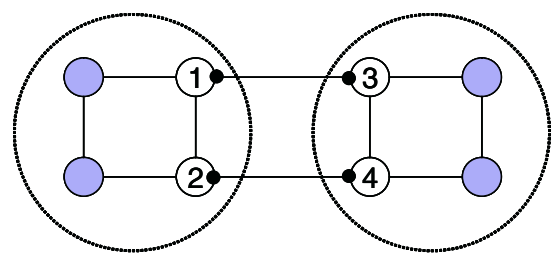

(a) Link-based network partition

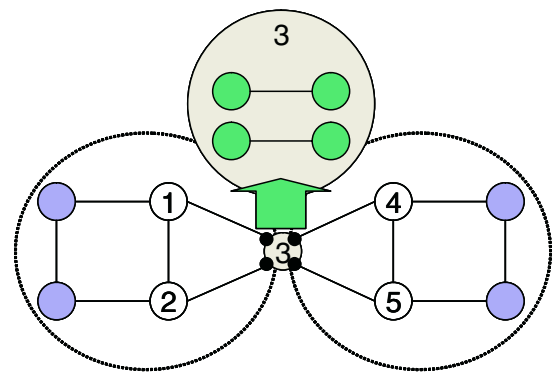

(b) Node-based network partition

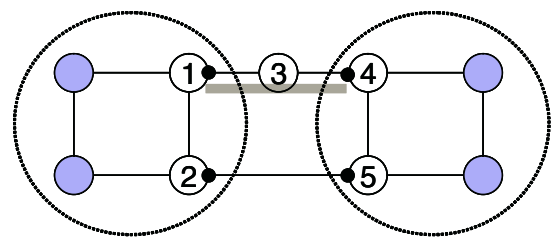

(c) Hybrid network partition

Fig. 8. Path partitioning methods

kinds of backup path (intra sub-network backup path and inter sub-networks backup path) [6], [7].

However, physical network based path partitioning is more useful in both transit network and multi-AS networks. So in this paper, we use network based partitioning method to implement the proposed segment restoration scheme.

\subsection{Network Partitioning}

Since restoration performance depends on the size and the topology of subnetworks, efficient network partitioning scheme is essential to enhance the restoration performance.

So we make several network partitioning rules as follows:

- Rule 1: Sub-network should have at least two edge nodes for connecting its adjacent sub-networks. This is required for the inter segments fault restoration; if there is just one node that connects two sub-networks, the inter segments fault cannot be restored. 
- Rule 2: A link or a node failure within a sub-network must be restored in its sub-network. The link connectivity degree of all nodes within subnetwork should be greater than or equal to 2. Mesh topology provides better restoration capability than star or hub and tree topology.

The Rule 1 is an essential requirement to perform the inter segment restoration and the Rule 2 is for the intra segment restoration [6], [7].

Network partitioning is achieved according to above two rules as follows:

- Link-based network partitioning: Basic partitioning rule of the proposed restoration scheme. Sub-networks are divided by links as shown in Fig. 8(a). Inter sub-network restoration is triggered when link 1-3 or link 2-4 is broken.

- Node-based network partitioning: If it is impossible to divide a network using link-based partitioning, sub-networks can be divided by node as shown in Fig. 8(b). In this case, we assume that a node consists of sub-nodes (ports) and sub-links. So, node-based network partitioning can be concerned a special subset of link-based network partitioning. In this case, inter sub-networks restoration is triggered when a fault occurs in the node 3 .

- Hybrid network partitioning: When it is impossible to divide using nodebased partition or link-based partition, hybrid network partitioning method can be used. In Fig. 8(c), node 3 excludes from two sub-networks; node 3 can be concerned as a link. The hybrid network partitioning is also the extension of link-based partitioning. When a fault occurs at link 1-3, link 3-4, link 2-5 or node 3 , inter sub-networks restoration is triggered

Generally, all three partitioning methods can be used in a large size transit network. Basically, link-based partitioning (which is our basic partitioning method) is derived from multi-AS networks.

\subsection{Backup Path Provisioning Rules for Inter-segments Restoration}

In our segment restoration schemes, faults are classified as intra sub-network fault that occurs in a sub-network, and inter sub-network fault that occurs between two sub-networks. After a working path is established, intra segment backup paths are established in each sub-network for intra segment restoration. Inter segment backup paths are established after each intra segment backup paths are established. We select the path with lowest cost among the four intersegment backup paths that are calculated by the four bridge methods.

Backup path provisioning methods for inter segment restoration is classified into four types: using two backup nodes, using a backup and a working node, using a working and a backup node, and using two working nodes.

- B2B(Backup node to Backup node) bridge: An inter segment backup path is established using two backup nodes as shown in Fig. 9(a).

- B2W(Backup node to Working node) bridge: An inter segment backup path is established using a backup node and a working node as shown in Fig. 9(b).

- W2B(Working node to Backup node) bridge: An inter segment backup path is established using a working node and a backup node as shown in Fig. 9(c). 


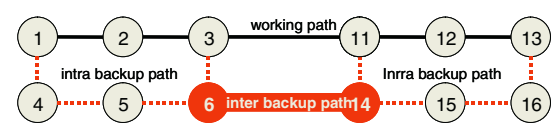

(a) B2B bridge

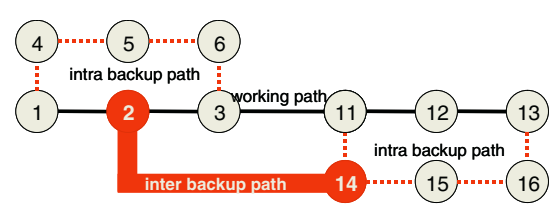

(c) W2B bridge

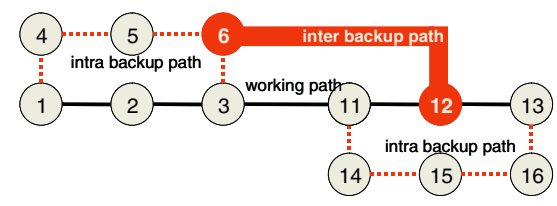

(b) B2W bridge

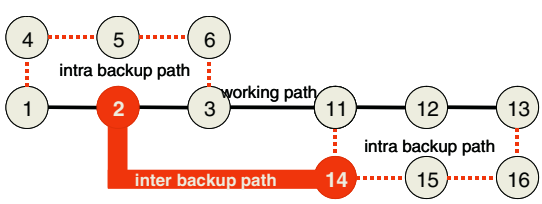

(d) W2W bridge

Fig. 9. Bridge methods for inter sub-network restoration

- W2W(Working node to Working node)bridge:Fig. 9(d) shows an inter segment backup path using two working nodes, which is the opposite case of using two backup nodes.

\section{Simulation and Evaluation}

In this section, we analyze and evaluate the performance of the proposed segment restoration scheme for its restoration time and resource requirements compared with other restoration schemes. We measure the average restoration time and backup resource usage for various working paths that have different hop length in two U.S. sample networks as shown in Fig. 10. We make the following assumptions for the simulation.

- All working paths and backup paths are established along its shortest path.

- Backup path should not belong to same SRLG(Shared Risk Link Group) of the working path

\subsection{Evaluation of Restoration Time}

To compare restoration performance, we use two restoration performance factors: restoration time and backup resource capacity. Restoration time is the most important factor for comparing restoration performance and backup resource capacity is also important to calculate network installation capacity. Restoration time is determined by the sum of fault detection time, fault notification propagation delay time and node processing time as (1) [8].

$$
T_{\text {restoration }}=T_{\text {detection }}+T_{\text {propagation delay }}+T_{\text {node processing }} .
$$

Here, $T_{\text {restoration }}$ is total restoration time, $T_{\text {detection }}$ is fault detection time, $T_{\text {propagation delay }}$ is propagation delay time and $T_{\text {node }}$ processing is message processing time in a node. 


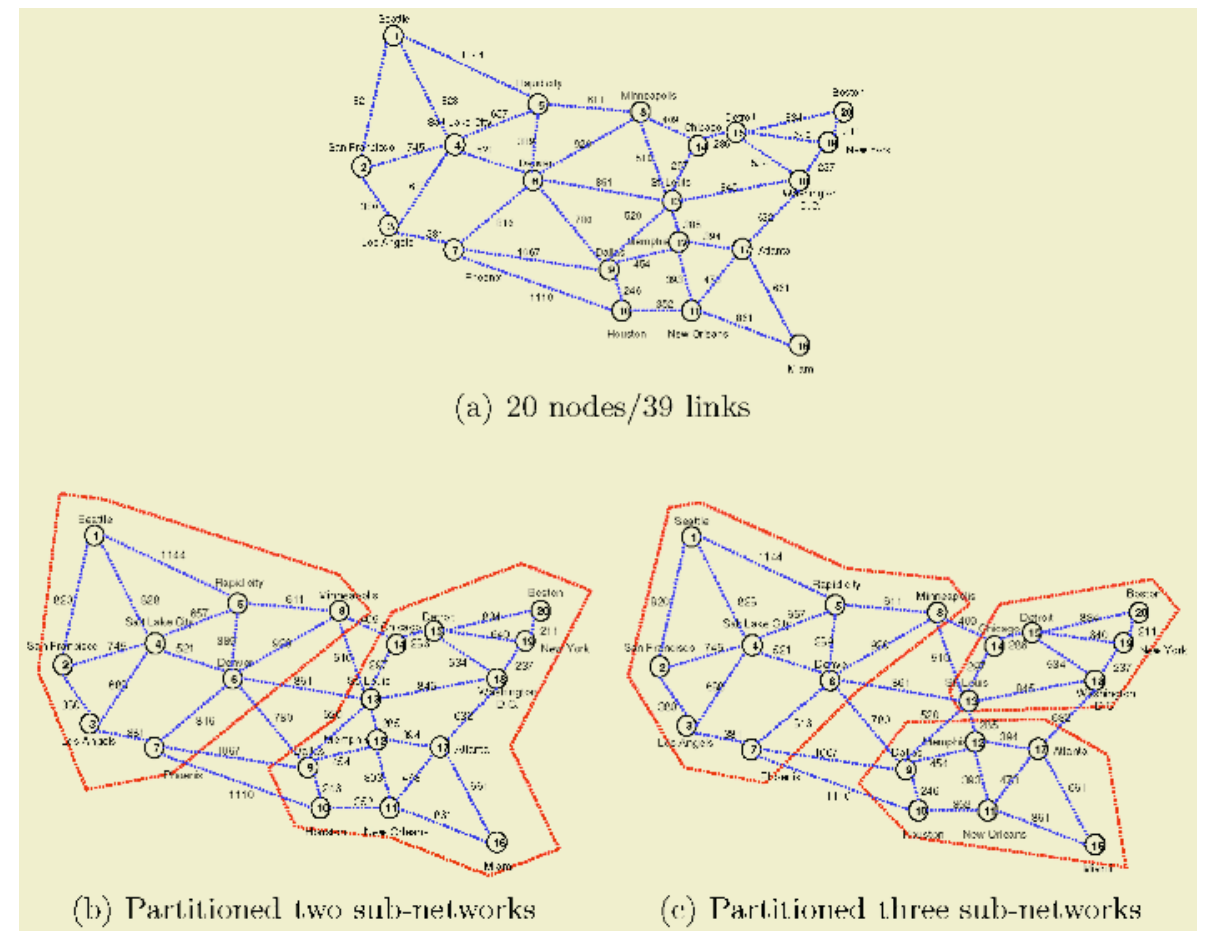

Fig. 10. U.S. sample network

For the simulation, we assume $T_{\text {detection }}$ sets to $10 \mathrm{~ms}$ and $T_{\text {node processing }}$ sets to $1 \mathrm{~ms}$ per node [9]. $T_{\text {propagation delay }}$ is calculated using the physical distance of links. So, propagation distance and the number of nodes that are passed by fault notification messages determine restoration time. To reduce restoration time, it is the best way to reduce transfer delay time and the number of nodes that are passed by fault notification messages.

Fig. 11 shows the restoration time according to the size of sub-networks and restoration schemes. In the proposed segment restoration scheme, the restoration time depends on the number of sub-networks. From Fig. 11, we can find that the restoration time of segment $\mathrm{x} 3$ is less than segment $\mathrm{x} 2$. So we can conclude when a network is divided into smaller sub-networks, the restoration performance can be improved

The restoration time of the segment restoration scheme is shorter than the path restoration scheme, but longer than the link restoration scheme. We assume only link failure in this simulation because the link restoration scheme can't restore any node failure and we can't measure the restoration time of the link restoration scheme when a node failure occurs. So the proposed segment restoration has the shortest restoration time in the mixed fault environment such as link failure, node failure and multiple failures. 


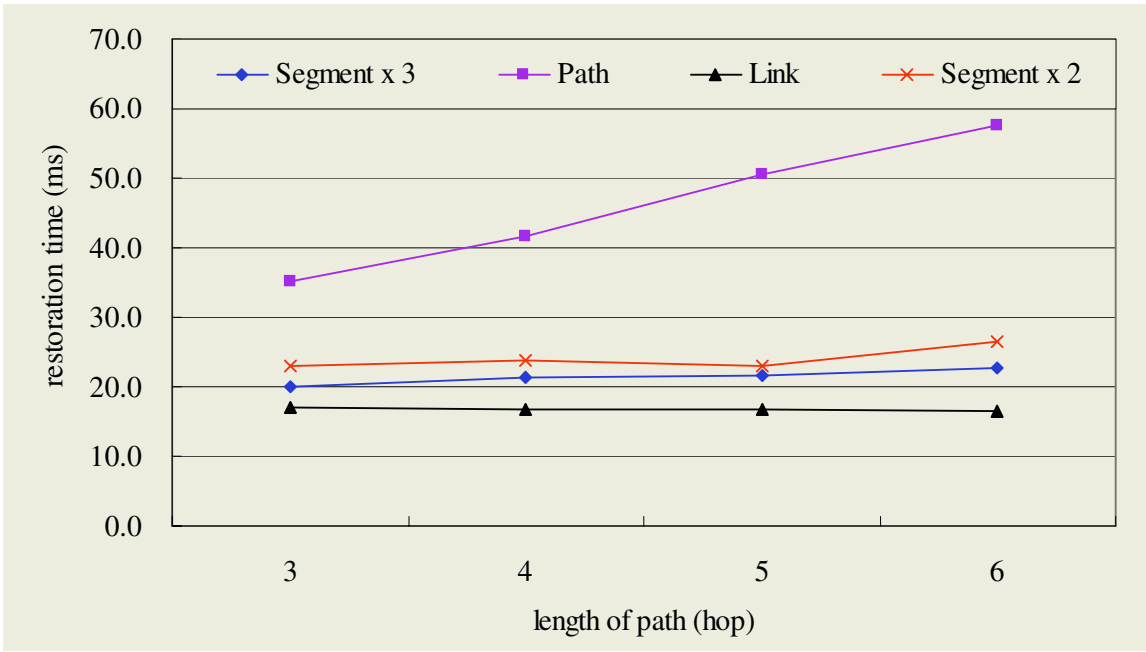

Fig. 11. Restoration time

\subsection{Evaluation of Restoration Restoration Capacity}

To compare backup resource capacity, we measure the ratio of the required resource of backup paths to working paths using (2).

$$
\text { Backup resource capacity }=\frac{\text { total bandwidth of the backup path }}{\text { total bandwidth of the working path }} * 100(\%) .
$$

Fig. 12 shows backup resource requirement according to the size of subnetworks and restoration schemes. In the proposed segment restoration scheme, the restoration resource capacity is increased when a network is divided into smaller sub-networks.

The path restoration scheme prepares backup resource for a whole path, but the link restoration scheme and the proposed segment restoration scheme prepares backup resource for every link or sub-network respectively; therefore more resource is required compared with the end-to-end path restoration. From the result, we can see that the path restoration scheme requires the least backup resource, while the proposed nt restoration requires more backup resource, but less than link restoration scheme. We summarize restoration performance as a function of the size of sub-network and restoration schemes in Table II.

The link restoration scheme has the shortest restoration time in this simulation, but it can't restore any node failure. So the proposed segment restoration has the shortest restoration time when link and node failure occur. The path restoration scheme has the smallest backup resource capacity compared with other restoration schemes but has the longest restoration time. Path restoration scheme can't guarantee quality of service (QoS) because of slow restoration time, so it is not adequate restoration scheme for high-speed networks such as MPLS and WDM. 


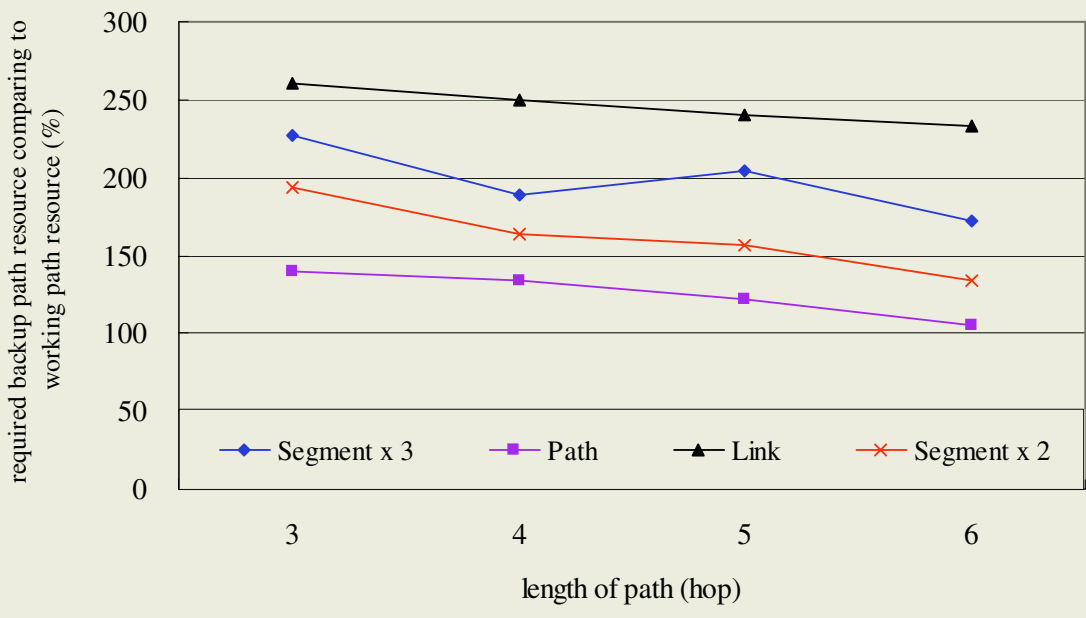

Fig. 12. Required backup resource

Table 1. Comparison of Restoration Performance

\begin{tabular}{cccc}
\hline & Restoration Time & Required Backup Resource \\
\hline \hline \multirow{2}{*}{ Proposed restoration } & Segment x 2 & $24.0 \mathrm{~ms}$ & $162 \%$ \\
\cline { 2 - 4 } & Segment x 3 & $21.4 \mathrm{~ms}$ & $198 \%$ \\
\hline Link Restoration & $16.8 \mathrm{~ms}$ & $250 \%$ \\
\hline Path Restoration & $46.2 \mathrm{~ms}$ & $132 \%$ \\
\hline \hline
\end{tabular}

\section{Conclusion}

Most restoration schemes for telecommunication network are based on link-based restoration or path-based restoration. The link restoration scheme has shortest restoration time, but lowest backup resource capacity. The path restoration scheme has opposite characteristic. It is difficult to satisfy both fast restoration speed and high resource utilization. But high-speed networks, such as MPLS network, require traffic engineering for optimized resource utilization. So restoration schemes for high-speed network must have fast restoration functions and efficient backup resource management functions. The proposed segment restoration is based on network partitioning that divides a large network into several small sub-networks. Because most faults are restored in sub-networks, fault restoration time is reduced obviously. And backup resource capacity is also less than the link restoration scheme.

From the simulation results, we verified that the segment restoration scheme has advantages in both restoration time and backup resource capacity. The proposed segment restoration can provide a good restoration performance for highspeed transport networks. 


\section{References}

1. IETF mpls working group, "Multi-protocol Label Switching Architecture," IETF Internet Draft, draft-ietf-mpls-arch-05,(1999).

2. ETRI, The Technical Introduction on Internet Service based on ATM, (1999).

3. Pin-Han Ho and Hussein. T. Mouftah, "A Framework for Service-Guaranteed Shared Protection in WDM Mesh Networks," IEEE Communication Magazine, (2002).

4. C. Ou, H. Zang, and B Mukherjee, "Sub-path protection for Scalability and Fast Recovery in WDM Mesh Networks," Proc. OFC2002, (2002).

5. D. Xu, Y. Xiong, and C. Qiao, "Protection with Multi-Segments(PROMISE) in Networks with Shared Risk Link Group(SRLG)," 40th Annual Allerton Conference on Communication, Control, and Computing, (2002).

6. Hae-Joon Shin, Jae-Jun Jang and Young-Tak Kim, "Subnetwork-based Segment Restoration for fast fault Recovery in the MPLS network," Journal of KICS, Vol. 27, No. 11C, (2002).

7. Hae-Joon Shin, Ryung-Min Kim, Young-Tak Kim, "Network Fault Recovery Algorithm based on Segment Automatic Restoration Scheme," Journal of KISS, Vol. 30, No. 3, (2003).

8. ITU-T Recommendation M.495, Maintenance : International Transmission System, (1993).

9. J. Anderson, B. T. Doshi, S. Dravida, and P. Harshavardhana, "Fast Restoration of ATM Networks," IEEE J. Selected Area in Comm., (1994)128-138. 